\title{
Assistance extracorporelle au cours du syndrome de détresse respiratoire aiguë (chez l'adulte et l'enfant, à l'exclusion du nouveau-né). Conférence de consensus organisée par la Société de réanimation de langue française
}

\author{
Extracorporeal Life Support for Patients with Acute Respiratory Distress Syndrome \\ (Adult and Paediatric). Consensus Conference Organized by the French Intensive Care Society
}

\author{
C. Richard $\cdot$ L. Argaud $\cdot$ A. Blet $\cdot$ T. Boulain $\cdot$ L. Contentin $\cdot$ A. Dechartres $\cdot$ J.-M. Dejode $\cdot$ L. Donetti $\cdot$ M. Fartoukh $\cdot$ \\ D. Fletcher $\cdot$ K. Kuteifan $\cdot$ S. Lasocki $\cdot$ J.-M. Liet $\cdot$ A.-C. Lukaszewicz $\cdot$ H. Mal $\cdot$ E. Maury $\cdot$ D. Osman $\cdot$ H. Outin $\cdot$ \\ J.-C. Richard $\cdot$ F. Schneider $\cdot$ F. Tamion
}

(C) SRLF et Springer-Verlag France 2014

\author{
C. Richard $(\bowtie)$ \\ Service de réanimation médicale, hôpitaux universitaires \\ Paris-Sud, AP-HP, hôpital de Bicêtre, EA-4533, \\ université Paris-Sud, \\ F-94270 Le Kremlin-Bicêtre, France \\ Président du jury. \\ e-mail : christian.richard@bct.aphp.fr

\section{Argaud} \\ Service de réanimation médicale, hospices civils de Lyon, \\ groupement hospitalier Édouard-Herriot, F-69437 Lyon, France \\ Jury.
}

\section{A. Blet}

Département d'anesthésie-réanimation, centre de traitement des brulés, AP-HP, hôpitaux universitaires

Saint-Louis-Lariboisière-Fernand-Widal, hôpital Saint-Louis,

F-75010 Paris, France

Groupe bibliographique.

\section{T. Boulain}

Service de réanimation polyvalente, CHR d'Orléans,

hôpital de La Source, F-45067 Orléans, France

Jury.

\section{Contentin}

Service de réanimation polyvalente, CHRU de Tours,

hôpital Bretonneau, F-37000 Tours, France

Groupe bibliographique.

\section{A. Dechartres}

Inserm U1153, équipe « Méthodes en évaluation thérapeutique des maladies chroniques ", centre de recherche épidémiologie et biostatistique, centre Cochrane français, Hôtel-Dieu,

F-75004 Paris, France

Groupe bibliographique.

\section{J.-M. Dejode}

Réanimation pédiatrique, CHU de Nantes, hôpital Mère-Enfant, F-40000 Nantes, France

Jury.

\section{Donetti}

Service de réanimation, $\mathrm{CH}$ de Le Raincy-Montfermeil,

F-93370 Montfermeil, France

Comité d'organisation.

M. Fartoukh

Unité de réanimation médicochirurgicale, AP-HP, hôpitaux universitaires Est-Parisien, hôpital Tenon, F-75020 Paris, France Jury.

\section{Fletcher}

Département d'anesthésie, hôpitaux universitaires Paris

Île-de-France Ouest, AP-HP, hôpital Raymond-Poincaré,

F-92380 Garches, France

Comité d'organisation.

K. Kuteifan

Service de réanimation médicale, hôpital Emile-Muller,

F-68070 Mulhouse, France

Comité d'organisation.

\section{S. Lasocki}

Pole d'anesthésie-réanimation, CHU d'Angers, LUNAM Université, université d'Angers, F-49000 Angers, France

Jury.

J.-M. Liet

Réanimation pédiatrique, CHU de Nantes, hôpital Mère-Enfant,

F-40000 Nantes, France

Jury.

A.-C. Lukaszewicz

Département d'anesthésie-réanimation, de réanimation chirurgicale et postopératoire, AP-HP, groupe hospitalier Saint-Louis-Lariboisière-Fernand-Widal, hôpital Lariboisière,

F-75010 Paris, France

Jury.

H. Mal

Service de pneumologie, hôpitaux universitaires Paris-Nord-Val-deSeine, AP-HP, hôpital Bichat, F-75018 Paris, France Jury. 


\section{E. Maury}

Service de réanimation médicale, AP-HP,

hôpitaux universitaires Est-Parisien, hôpital Saint-Antoine,

F-75020 Paris, France

Jury.

\section{Osman}

Service de réanimation médicale, hôpitaux universitaires Paris-Sud, AP-HP, hôpital de Bicêtre, F-94270 Le Kremlin-Bicêtre, France Comité d'organisation.

\section{H. Outin}

Service de réanimation médicochirurgicale, centre hospitalier intercommunal de Poissy-Saint-Germain-en-Laye, F-78300 Poissy, France

Jury.

\section{J.-C. Richard}

Service de réanimation médicale, hospices civils de Lyon, hôpital de la Croix-Rousse, F-69004 Lyon, France

Comité d'organisation.

F. Schneider

Service de réanimation médicale, hôpitaux universitaires de Strasbourg et faculté de médecine, université de Strasbourg, hôpital de Hautepierre, Strasbourg, France Jury.

\section{F. Tamion}

Service de réanimation médicale, Inserm U1096, IRIB, CHU Charles-Nicolle, université de Rouen, F-76031 Rouen, France Jury.
Résumé En 2009, à l'occasion de l'épidémie de grippe H1N1, la description de formes très sévères de syndrome de détresse respiratoire aiguë (SDRA) s'est accompagnée d'un renouveau de l'utilisation des techniques d'assistance respiratoire extracorporelle, aboutissant à considérer qu'elles avaient une place dans l'algorithme de prise en charge du SDRA. La rareté des indications et l'absence de preuves formelles de l'impact sur le pronostic des patients ont amené la Société de réanimation de langue française (SRLF) à organiser en partenariat avec la Société française d'anesthésie et de réanimation (Sfar), la Société de pneumologie de langue française (SPLF), le Groupe francophone de réanimation et d'urgences pédiatriques (GFRUP), la Société française de perfusion (Sofraperf), la Société française de chirurgie thoracique et cardiovasculaire (SFCTV) et la Société espagnole de soins intensifs (SEMICYUC), une conférence de consensus sur l'assistance extracorporelle au cours du SDRA. Un jury, composé de réanimateurs, a ainsi élaboré 65 recommandations correspondant aux cinq questions qui leur étaient posées : 1) quelles sont les techniques disponibles ? 2) Quels patients relèvent d'une assistance extracorporelle ? 3) Comment réaliser l'assistance extracorporelle ? 4) Quand et comment arrêter une assistance extracorporelle ? 5) Quelle organisation proposer ? La rédaction de ces recommandations a pris en compte les données de la littérature scientifique, analysée selon la méthode GRADE, ainsi que les données recueillies à l'occasion d'une réunion rassemblant experts et professionnels.

Mots clés Extracorporeal life support (ECLS) - Extracorporeal membrane oxygenation (ECMO) - Extracorporeal $\mathrm{CO}_{2}$ removal $\left(\mathrm{ECCO}_{2} \mathrm{R}\right) \cdot$ Syndrome de détresse respiratoire aiguë $(\mathrm{SDRA}) \cdot$ Ventilation protectrice
Abstract The H1N1 influenza epidemics in 2009 led a substantial number of people to develop severe acute respiratory distress syndrome and refractory hypoxemia. In these patients with severe acute respiratory distress syndrome, extracorporeal membrane oxygenation was used as rescue oxygenation therapy. Several randomized clinical trials and observational studies suggested that extracorporeal membrane oxygenation associated with protective mechanical ventilation could improve outcome, but its efficacy remains uncertain. Organized by the Société de réanimation de langue française (SRLF) in conjunction with the Société française d'anesthésie et de réanimation (Sfar), the Société de pneumologie de langue française (SPLF), the Groupe francophone de réanimation et d'urgences pédiatriques (GFRUP), the Société française de perfusion (Sofraperf), the Société française de chirurgie thoracique et cardiovasculaire (SFCTV) et the Société espagnole de soins intensifs (SEMICYUC), a consensus conference was held in December 2013 and a jury of 13 members wrote 65 recommendations to answer the five following questions regarding the place of extracorporeal life support for patients with acute respiratory distress syndrome: 1) What are the available techniques? 2) Which patients could benefit from extracorporeal life support? 3) How to perform extracorporeal life support? 4) How and when to stop extracorporeal life support? 5) Which organization should be recommended? To write the recommendations, evidence-base medicine (GRADE method), expert panel opinions, and shared decisions taken by all the thirteen members of the jury of the consensus conference were taken into account.

Keywords Extracorporeal life support (ECLS) · Extracorporeal membrane oxygenation (ECMO) - Extracorporeal $\mathrm{CO}_{2}$ removal $\left(\mathrm{ECCO}_{2} \mathrm{R}\right) \cdot$ Acute respiratory distress syndrome (ARDS) - Protective ventilation 
Président du jury :

SRLF : Christian Richard (Le Kremlin-Bicêtre)

\section{Membres du jury :}

SRLF :

Laurent Argaud (Lyon)

Thierry Boulain (Orléans)

Eric Maury (Paris)

Hervé Outin (Poissy)

Francis Schneider (Strasbourg)

Fabienne Tamion (Rouen)

Sfar :

Sigismond Lasocki (Angers)

Anne-Claire Lukaszewicz (Paris)

SPLF:

Muriel Fartoukh (Paris)

Hervé Mal (Paris)

GFRUP:

Jean-Marc Dejode (Nantes)

Jean-Michel Liet (Nantes)

\section{Conseillers scientifiques :}

SRLF:

Claude Guérin (Lyon)

SEMICYUC:

Antonio Artigas (Sabadell)

\section{Comité d'organisation}

SRLF :

Laurence Donetti, présidente (Montfermeil)

Khaldoun Kuteifan (Mulhouse)

David Osman (Le Kremlin-Bicêtre)

Jean-Christophe Richard (Lyon)

Sfar :

Dominique Fletcher (Paris)

\section{Chargés de bibliographie :}

SRLF :
Alice Blet (Paris)
Laetitia Contentin (Tours)
Centre Cochrane français :
Agnès Dechartres (Paris)

\section{Experts :}

Désignés par la SRLF :

Laurent Brochard (Genève)

Gilles Capellier (Besançon)

Alain Combes (Paris)

Luciano Gattinoni (Milan)

Luc-Marie Jacquet (Bruxelles)

François Lemaire (Paris)

Bruno Mégarbane (Paris)
Giles Peek (Leicester)

Antoine Roch (Marseille)

Désigné par la Sfar :

Olivier Bastien (Lyon)

Désignés par la SFCTCV :

Guillaume Lebreton (Paris)

Pascal Leprince (Paris)

Désigné par le GFRUP :

Sylvain Renolleau (Paris)

Désignée par la Sofraperf :

Laurence Omnes (Paris)

\section{Introduction}

La mortalité du syndrome de détresse respiratoire aiguë (SDRA) reste élevée dans ses formes sévères telles que définies lors de la récente conférence de consensus de Berlin [1]. Une des explications retenues à l'appui de ce constat est l'existence d'un groupe de patients qui demeurent sévèrement hypoxémiques en dépit d'une prise en charge ventilatoire reposant sur les préconisations internationales actuelles, comportant en particulier le recours à un volume courant compris entre 4 et $8 \mathrm{ml} / \mathrm{kg}$, à un recrutement alvéolaire par l'utilisation de hauts niveaux de pression expiratoire positive, au décubitus ventral et à la curarisation initiale [2]. Chez ces malades s'ajoute fréquemment une acidose respiratoire, en rapport avec la sévérité du trouble de la compliance thoracopulmonaire, obligeant parfois à réduire encore le volume courant et/ou le niveau de pression expiratoire positive afin de maintenir une pression de plateau inférieure à $30 \mathrm{cmH}_{2} \mathrm{O}$.

Dans ces situations extrêmement sévères a été proposée depuis les années 1980 l'utilisation des techniques d'assistance extracorporelle d'oxygénation (extracorporeal membrane oxygenation, ECMO) dans le but d'attendre la récupération fonctionnelle pulmonaire [3]. Deux raisons principales expliquent les très mauvais résultats et le rapport bénéfice/risque très défavorable des études initiales [3,4] : l'importance des complications, en particulier hémorragiques, et la poursuite de la ventilation mécanique selon des modalités qui, à l'époque, prenaient mal en compte les risques baro- et volotraumatiques pulmonaires.

Plus récemment, à l'occasion en particulier de l'épidémie de grippe $\mathrm{H} 1 \mathrm{~N} 1$, la description de formes très sévères et d'installation rapide de SDRA, à l'origine d'hypoxémie réfractaire, s'est accompagnée d'un renouveau de l'utilisation de ces techniques en association avec une ventilation protectrice [5-8]. À partir de la tenue de registres nationaux et internationaux ont été suggérés des résultats favorables avec les techniques d'assistance circulatoire extracorporelle utilisées précocement en cas d'hypoxémie sévère [5-8]. 
La mise à disposition large d'appareils d'assistance extracorporelle, le plus souvent à partir des centres disposant d'un service de chirurgie cardiaque et/ou thoracique entraînés à leur utilisation quotidienne dans des indications cardiologiques, a abouti à considérer que l'ECMO, en particulier veinoveineuse (ECMO VV), avait désormais sa place dans l'algorithme de prise en charge du SDRA $[2,9,10]$.

La rareté des indications, l'absence de preuves formelles de l'impact sur le pronostic des patients, associées à des risques connus potentiellement graves, à une charge en soins considérable, à un rapport coût/bénéfice inconnu et à l'indispensable nécessité d'une formation théorique et pratique des personnels médicosoignants, ont amené la Société de réanimation de langue française (SRLF) à décider de la tenue d'une conférence de consensus destinée à préciser les modalités d'utilisation de ces techniques d'assistance extracorporelle. Les différentes disciplines contribuant à la mise en ouvre des techniques d'assistance extracorporelle au cours du SDRA étaient représentées à travers la participation de la Société française d'anesthésie et de réanimation (Sfar), la Société de pneumologie de langue française (SPLF), le Groupe francophone de réanimation et d'urgences pédiatriques
(GFRUP), la Société française de perfusion (Sofraperf), la Société française de chirurgie thoracique et cardiovasculaire (SFCTV) et la Société espagnole de soins intensifs (SEMICYUC).

Prenant également en compte le développement des techniques d'épuration du $\mathrm{CO}_{2}$ (extracorporeal $\mathrm{CO}_{2}$ removal, $\left.\mathrm{ECCO}_{2}-\mathrm{R}\right)[11,12]$ dont l'implantation en France paraît se faire à ce jour sans la mise en place d'une démarche coordonnée et d'une quelconque évaluation, la SRLF a fixé au jury de cette conférence l'objectif d'établir des recommandations à partir des cinq questions suivantes :

- quelles sont les techniques disponibles?

- Quels patients relèvent d'une assistance extracorporelle ?

- Comment réaliser l'assistance extracorporelle ?

- Quand et comment arrêter une assistance extracorporelle ?

- Quelle organisation proposer?

La rédaction de ces recommandations a pris en compte les données de la littérature scientifique rassemblées par un groupe bibliographique encadré par un membre du Centre " Cochrane Français », celles recueillies auprès des experts et des participants à l'occasion d'une réunion publique et l'opinion du jury permettant ainsi, dans la majorité des cas, de proposer des recommandations consensuelles (Tableau 1).

Tableau 1 Recommandations du jury pour l'assistance extracorporelle au cours du SDRA

\section{1 : Quelles sont les techniques d'assistance extracorporelle disponibles?}

1.1 Les techniques d'assistance extracorporelle à haut débit permettent au cours d'une ventilation conventionnelle et protectrice d'améliorer l'oxygénation en cas d'hypoxémie réfractaire et/ou de corriger une hypercapnie

1.2 Les techniques d'assistance extracorporelle à haut débit permettent de mettre en œuvre des stratégies de ventilation « ultraprotectrice»

1.3 Les techniques d'épuration du $\mathrm{CO}_{2}$ à faible débit permettent de mettre en œuvre des stratégies de ventilation « ultraprotectrice » 1.4 Au cours de l'ECMO, le débit obtenu au niveau de la canule de drainage est un déterminant essentiel de l'efficacité de l'oxygénation 1.5 L'insertion et l'utilisation des canules coaxiales exposent à un risque accru de perforation myocardique

1.6 Au cours de l'ECMO, le choix du site d'insertion et des diamètres des canules doit permettre de générer un débit d'assistance adapté aux objectifs thérapeutiques

1.7 Chez l'adulte, au cours de l'ECMO veinoveineuse, la configuration fémorojugulaire interne doit être favorisée pour permettre de générer un débit d'assistance adapté aux objectifs thérapeutiques (RC)

1.8 Au cours de l'ECMO, l'utilisation de pompes centrifuges non occlusives doit être favorisée (RC)

1.9 Au cours de l'ECMO, l'utilisation d'oxygénateurs à membrane en polyméthylpentène doit être favorisée (RC)

\section{2 : Quels patients relèvent d'une assistance extracorporelle au cours du SDRA ?}

2.1 Les indications de l'ECMO doivent reposer sur une décision collégiale et multidisciplinaire, renseignée dans le dossier médical (RC) 2.2 Au cours du SDRA, il faut discuter les indications d'ECMO au cas par cas, en prenant en compte le rapport bénéfice/risque

2.3 L'information et la recherche du consentement du patient, à défaut de la personne de confiance, sont des préalables obligatoires à la mise en œuvre de l'ECMO au cours du SDRA

2.4 Lorsque le patient est hors d'état d'exprimer sa volonté, et en dehors des situations d'urgence, l'information des proches est un préalable obligatoire à la mise en œuvre de l'ECMO au cours du SDRA

2.5 La réversibilité prévisible des lésions pulmonaires et l'absence de toute autre limitation thérapeutique constituent un prérequis indispensable à l'utilisation de l'ECMO (RC)

2.6 Devant une forme sévère de SDRA, il ne faut pas recourir à l'ECMO tant qu'une stratégie de ventilation protectrice comportant, lorsqu'il est possible, le recours au décubitus ventral n'a pas été mise en place

2.7 Chez l'enfant, lorsque la $\mathrm{PaO}_{2}$ n'est pas disponible, il est possible d'évaluer la gravité du SDRA en utilisant le ratio $\mathrm{SpO}_{2} / \mathrm{FiO}_{2}(\mathrm{RC})$ 
Tableau 1 (suite)

2.8 Parmi les techniques d'assistance extracorporelle, l'ECMO veinoveineuse est la technique de référence au cours du SDRA sévère 2.9 Il ne faut pas, en l'état actuel des connaissances, utiliser les techniques d'épuration du $\mathrm{CO}_{2}$ à faible débit $\left(\mathrm{ECCO}_{2}-\mathrm{R}\right)$ au cours du SDRA

2.10 Les techniques d'épuration du $\mathrm{CO}_{2}$ à faible débit $\left(\mathrm{ECCO}_{2}-\mathrm{R}\right)$ doivent faire l'objet d'une évaluation dans le cadre d'essais cliniques (RC)

$2.11 \mathrm{Il}$ faut envisager le recours à l'ECMO VV si le rapport $\mathrm{PaO}_{2} / \mathrm{FiO}_{2}$ est inférieur à $50 \mathrm{mmHg}$ sous une FiO $2=1$, pendant au moins 3 heures en dépit d'une stratégie de ventilation protectrice (comportant le recours au décubitus ventral) [RC]

$2.12 \mathrm{Il}$ faut engager une réflexion sur le recours à l'ECMO VV si le rapport $\mathrm{PaO}_{2} / \mathrm{FiO}_{2}$ est inférieur à $80 \mathrm{mmHg}$ sous une $\mathrm{FiO}=1$, pendant plus de 6 heures en dépit d'une stratégie de ventilation protectrice (comportant le recours au décubitus ventral) [RC]

$2.13 \mathrm{Il}$ faut engager une réflexion sur le recours à l'ECMO VV si, associée à une stratégie de ventilation protectrice (comportant le recours au décubitus ventral), existe une acidose respiratoire avec un $\mathrm{pH}<7,20$ pendant plus de 6 heures (RC)

2.14 Il n'existe pas d'indication de l'ECMO VA au cours du SDRA lorsque la défaillance respiratoire est isolée. Un choc cardiogénique associé peut la faire discuter $(\mathrm{RC})$

2.15 Lorsqu'elle fait décider de l'ECMO, la présence d'un cœur pulmonaire aigu au cours du SDRA n'est pas une indication obligatoire à l'ECMO VA (RC)

2.16 L'impossibilité de recourir à une anticoagulation est une contre-indication classique à l'ECMO (RC)

2.17 Le rapport bénéfice/risque de l'ECMO au cours du SDRA doit être considéré comme défavorable en cas de 1) lésions intracrâniennes hémorragiques ou potentiellement hémorragiques ; 2) coma dans les suites d'un arrêt cardiaque ; 3) SDRA dont la durée de ventilation mécanique excède 7 jours ; 4) immunodépression sévère ; 5) syndrome de défaillance multiviscérale $(\mathrm{SOFA}>15)[\mathrm{RC}]$

\section{3 : Comment réaliser l'assistance extracorporelle au cours du SDRA ?}

3.1 Le montage, la pose et la gestion quotidienne de l'ECMO doivent être formalisés. Dans ce but, l'utilisation de « check-lists » est recommandée $(\mathrm{RC})$

3.2 Pour la mise en place et la gestion d'une ECMO, la présence d'un personnel médicosoignant formé au montage du circuit est recommandée $(\mathrm{RC})$

3.3 Au cours de l'ECMO, un réglage de la ventilation mécanique permettant de limiter au maximum le niveau de pression de plateau tout en administrant un niveau minimum de PEP est nécessaire

3.4 Lors de la canulation percutanée, la possibilité de recourir en urgence à une compétence chirurgicale vasculaire et thoracique doit être organisée (RC)

3.5 Pour l'insertion percutanée des canules d'ECMO, il faut réaliser la ponction vasculaire par échoguidage

3.6 Le bon positionnement des canules d'ECMO doit être contrôlé par échographie et radiographie de thorax (RC)

3.7 Pour une efficacité optimale de l'oxygénation par ECMO VV, le débit de la pompe à sang doit être $\geq 60 \%$ du débit cardiaque théorique

3.8 La fraction en oxygène délivrée par le circuit extracorporel $\left(\mathrm{F}_{\mathrm{ec}} \mathrm{O}_{2}\right)$ doit permettre d'obtenir une $\mathrm{SaO}_{2} \geq 88 \%$

3.9 Le débit de balayage doit permettre d'obtenir une $\mathrm{PaCO}_{2}$ comprise entre 30 et $40 \mathrm{mmHg}$

3.10 Une anticoagulation par héparine non fractionnée doit être instituée avec pour objectif un TCA entre 1,2 et 1,5 × témoin (ou une activité anti-Xa entre 0,2 et $0,4 \mathrm{UI} / \mathrm{ml})[\mathrm{RC}]$

3.11 En cas de saignement significatif, l'anticoagulation doit être diminuée ou totalement arrêtée. La poursuite de l'ECMO doit être discutée $(\mathrm{RC})$

3.12 Chez l'enfant, un monitorage précis de l'hémostase est nécessaire comportant notamment le thromboélastogramme et la mesure de l'activité anti-Xa (RC)

3.13 Au cours de l'ECMO, lorsque le débit de la pompe à sang chute malgré le maintien de la vitesse de rotation, une réduction de précharge ou une augmentation de postcharge doivent être recherchées

3.14 Lors de la mise en route d'une ECMO VV pour SDRA, l'aggravation ou la persistance d'une hypoxémie peut être secondaire à la levée de la vasoconstriction pulmonaire hypoxique, mais doit faire rechercher une cause mécanique (défaillance de l'oxygénateur, débit insuffisant, recirculation) [RC]

3.15 Lors de la réalisation d'une ECMO VV pour SDRA, l'aggravation d'une hypoxémie peut être secondaire à une aggravation pulmonaire, mais doit faire rechercher une cause mécanique (défaillance de l'oxygénateur, débit insuffisant, recirculation) [RC]

\section{4 : Quand et comment arrêter une circulation extracorporelle au cours du SDRA ?}

4.1 La question du sevrage de l'ECMO doit être posée de façon quotidienne (RC)

4.2 Concernant la poursuite de l'ECMO, la question de l'obstination déraisonnable doit être discutée au cours de réunions collégiales et multidisciplinaires (RC)

4.3 L'arrêt de l'ECMO est fortement recommandé lors de la survenue d'une complication cérébrale grave hémorragique ou embolique (RC) 4.4 Chez l'enfant, les modalités de sevrage de l'ECMO ne sont pas différentes de celles de l'adulte (RC)

4.5 La procédure de sevrage de l'ECMO comporte la recherche quotidienne de critères témoignant d'une récupération de la défaillance respiratoire ou cardiorespiratoire $(\mathrm{RC})$ 
Tableau 1 (suite)

4.6 Lors de la procédure de sevrage de l'ECMO, l'absence de cœur pulmonaire aigu doit être recherchée (RC)

4.7 L'arrêt de l'ECMO est décidé selon les résultats d'une procédure de sevrage formalisée de plusieurs heures (RC)

4.8 Le sevrage de l'ECMO VA impose le maintien d'un balayage sur l'oxygénateur (RC)

4.9 Au cours du SDRA, l'absence de sevrage possible d'une ECMO VA doit faire discuter la possibilité d'une conversion en ECMO VV (RC)

4.10 L'anticoagulation doit être arrêtée au moins 1 heure avant la décanulation (RC)

4.11 La décanulation peut être réalisée au bloc opératoire ou en réanimation ( $\mathrm{RC})$

4.12 La décanulation artérielle est toujours un geste chirurgical. La décanulation veineuse peut être médicale ou chirurgicale (RC)

4.13 Chez l'enfant, la décanulation veineuse ou artérielle est toujours chirurgicale (RC)

\section{5 : Quelle organisation proposer ?}

5.1 En regard de la qualité et de la sécurité des soins, une organisation territoriale structurée est indispensable à la prise en charge optimale des patients atteints de SDRA relevant de l'ECMO (RC)

5.2 En dehors de tout épisode épidémique, l'organisation sur le territoire national doit permettre la prise en charge d'au minimum 300 patients par an (RC)

5.3 La mise en place d'une activité d'ECMO doit s'inscrire dans un projet médical d'établissement (RC)

5.4 Il est nécessaire que, dans chaque région, soit identifié au minimum un centre référent possédant tous les moyens humains et matériels indispensables à la prise en charge d'un patient atteint de SDRA et à l'implantation et la conduite des techniques d'assistance extracorporelle : réanimation, chirurgie cardiaque et unité mobile d'assistance circulatoire (RC)

5.5 Les centres référents doivent disposer d'une unité mobile d'assistance circulatoire disponible 24 heures/24, à même d'intervenir dans tous les établissements de santé de la région concernée (RC)

5.6 Chaque service de réanimation doit colliger dans un registre national tous les patients pris en charge pour SDRA sévère (RC)

5.7 Chaque service de réanimation doit s'organiser par convention afin qu'au sein de chaque région la prise en charge des patients atteints de SDRA et relevant d'un traitement par ECMO soit assurée

5.8 Dans chaque région, au moins un service de réanimation compétent pour l'ECMO, appartenant au centre référent ou ayant contractualisé avec l'un d'entre eux, doit être identifié (RC)

5.9 Un service de réanimation compétent pour la pratique de l'ECMO dans le SDRA doit obligatoirement : 1) acquérir et entretenir des compétences spécifiques ; 2) compter dans son personnel médical au minimum deux médecins formés ; 3) disposer d’un recours en urgence à la chirurgie vasculaire et thoracique ; 4) mettre en œuvre un programme régulier de formation du personnel paramédical ; 5) formaliser les indications et en assurer la traçabilité ; 6) renseigner le registre SDRA sévère et 7) analyser, au moins une fois par an, à l'occasion de revues de morbidité-mortalité, l'ensemble des dossiers des patients traités par ECMO (RC)

5.10 Le maintien de la compétence en ECMO d'un service de réanimation risque de ne pas être assuré en deçà de dix indications annuelles d'ECMO

5.11 Pour l'enfant, une organisation interrégionale est nécessaire, organisée autour de centres référents pédiatriques (réanimation pédiatrique spécialisée), possédant tous les moyens humains et matériels indispensables à la prise en charge d'un patient atteint de SDRA et à l'implantation et la conduite des techniques d'assistance extracorporelle : réanimation, chirurgie cardiaque et unité mobile d'assistance circulatoire (RC)

5.12 Pour l'enfant, afin de permettre la réalisation de l'ECMO dans les meilleurs délais, le contact avec le centre référent pédiatrique (réanimation pédiatrique spécialisée) doit être précoce (RC)

$\mathrm{RC}$ : recommandation consensuelle.

\section{Méthodologie}

L'analyse de la littérature et la formulation des recommandations ont été conduites selon la méthode GRADE (Grade of recommendation assessment, development and evaluation) $[13,14]$. La recherche bibliographique a identifié les publications sans restriction de langue ni de date à partir des bases de données EMBASE, MEDLINE et The Cochrane Central Register of Controlled Trials [CENTRAL] (voir le supplément électronique). Les essais randomisés contrôlés, études de cohortes ou registres étaient inclus. Pour les publications antérieures à 1994, seuls les essais randomisés contrôlés étaient inclus.
La sélection des études pertinentes a été réalisée par deux personnes de manière indépendante en examinant successivement le titre, les résumés puis la totalité des textes si un article potentiellement approprié était identifié. Pour chaque étude jugée pertinente, le niveau de preuve a été évalué en fonction du type de l'étude (essai contrôlé randomisé, étude de cohorte, étude cas-témoin) et de sa qualité méthodologique. Les études sélectionnées ont ensuite été classées afin de regrouper celles qui évaluaient les différents critères de jugement prédéfinis, et un niveau de preuve global pour chacun de ces critères a été déterminé en prenant en compte le niveau de preuve des études individuelles. Un niveau global de preuve « fort » permettait de formuler une 
recommandation « forte » (il faut faire, ne pas faire, le jury recommande...). Un niveau global de preuve modéré, faible ou très faible aboutissait à l'écriture d'une recommandation dite « optionnelle » (il faut probablement faire ou probablement ne pas faire...).

Néanmoins, le type de formulation choisi n'était pas déterminé uniquement par le niveau global de preuve, mais pouvait être influencé par l'analyse du jury de la balance risque/bénéfice. Lorsque le choix de la formulation n'était pas consensuel, le président du jury procédait à un vote. Étant donné le thème général de la conférence et compte tenu de l'absence de preuve scientifique pour beaucoup des sujets traités, le jury a élaboré des « recommandations consensuelles » pouvant faire appel à un vote. Ces recommandations consensuelles sont signalées dans le texte par le sigle « RC » lorsqu'elles obtenaient le suffrage de l'ensemble du groupe. Enfin, le jury a souvent souhaité proposer de simples constats sans proposition d'actions. Le texte était alors rédigé en utilisant une formulation « formelle » ou « optionnelle».

\section{Quelles sont les techniques d'assistance extracorporelle disponibles ?}

\section{Définitions}

1.1 Les techniques d'assistance extracorporelle à haut débit (extracorporeal membrane oxygenation, ECMO) permettent au cours d'une ventilation conventionnelle et protectrice d'améliorer l'oxygénation en cas d'hypoxémie réfractaire et/ou de corriger une hypercapnie.

1.2 Les techniques d'assistance extracorporelle à haut débit (extracorporeal membrane oxygenation, ECMO) permettent de mettre en ouvre des stratégies de ventilation « ultraprotectrice ».

1.3 Les techniques d'épuration du $\mathrm{CO}_{2}$ à faible débit (extracorporeal $\mathrm{CO}_{2}$ removal, $\mathrm{ECCO}_{2}-\mathrm{R}$ ) permettent de mettre en ouvre des stratégies de ventilation « ultraprotectrice».

$\mathrm{Au}$ cours du SDRA, plusieurs techniques d'assistance extracorporelle permettent de répondre aux objectifs d'oxygénation ou d'épuration du $\mathrm{CO}_{2}$ du sang, mais aussi de limitation des lésions pulmonaires induites par la ventilation mécanique $[9,11,15,16]$. Ces suppléances, maintenues quelques jours à quelques semaines, permettent $\mathrm{d}$ 'attendre la récupération fonctionnelle pulmonaire en cas d'atteinte respiratoire réversible [6-8]. La correction de l'hypoxémie nécessite des débits d'assistance extracorporelle élevés de l'ordre de 3 à $7 \mathrm{l} / \mathrm{min}$. Une épuration efficace du $\mathrm{CO}_{2}$ peut être obtenue avec des débits d'assistance faibles de l'ordre de 500 à $1500 \mathrm{ml} / \mathrm{min}$ [17].
Assistance extracorporelle à haut débit (ECMO)

L'ECMO permet d'améliorer l'oxygénation en cas d'hypoxémie réfractaire aux techniques de ventilation conventionnelle, en utilisant une canulation veinoveineuse (ECMO VV) ou une canulation veinoartérielle (ECMO VA) [9]. Au cours de l'ECMO $V V$, le sang veineux est drainé par une canule veineuse insérée dans une grosse veine (jugulaire interne ou fémorale) et réinjecté après oxygénation dans ou à proximité immédiate de l'oreillette droite par une canule jugulaire interne ou fémorale. Le plus souvent, le drainage s'effectue au niveau veineux fémoral, et le retour au niveau jugulaire interne. L'ECMO VV peut être réalisée au moyen d'une canule coaxiale à double lumière ne nécessitant qu'un seul abord vasculaire [18]. Au cours de l'ECMO VA, le sang veineux est drainé par une canule insérée dans une grosse veine (jugulaire interne ou fémorale) et réinjecté après oxygénation par une canule artérielle fémorale (l'extrémité se situant alors dans l'aorte) ou axillaire [19].

Assistance extracorporelle à faible débit $\left(E C \mathrm{CO}_{2}-\mathrm{R}\right)$

$\mathrm{L}^{\prime} \mathrm{ECCO}_{2}-\mathrm{R}$ inclut la technique veinoveineuse d'épuration $\mathrm{du} \mathrm{CO}_{2}$ à faible débit (veno-venous $\mathrm{CO}_{2}$ removal, $\mathrm{VVCO}_{2}-\mathrm{R}$ ) et le shunt artérioveineux d'épuration du $\mathrm{CO}_{2}$ sans pompe (arterio-venous $\mathrm{CO}_{2}$ removal, $\mathrm{AVCO}_{2}-\mathrm{R}$ ) [20]. Elle permet une épuration du $\mathrm{CO}_{2}$ du sang et l'utilisation de stratégies de ventilation " ultraprotectrice », réduisant le volume courant et les pressions générées au niveau des alvéoles pulmonaires [12]. La technique de $\mathrm{VVCO}_{2}-\mathrm{R}$ nécessite un drainage veineux (jugulaire interne ou fémoral) et une réinjection dans le système veineux (jugulaire interne ou fémoral) après passage à travers un échangeur gazeux extrapulmonaire. Cette technique se rapproche de la technique d'hémofiltration continue, notamment du fait de l'utilisation de cathéters et non de canules [21]. Cette technique nécessite une pompe et un échangeur séparés ou intégrés dans une même unité. Les débits sont généralement faibles (300 à $1500 \mathrm{ml} / \mathrm{min}$ ). Les technologies les plus récentes permettent une élimination du $\mathrm{CO}_{2}$ plus efficace avec des surfaces de membrane moindres $[11,15]$. La technique d'AVCO ${ }_{2}-R$ utilise le débit sanguin du patient, qui doit être capable de générer un gradient de pression artérioveineux supérieur ou égal à $60 \mathrm{mmHg}$. L'existence d'un bas débit cardiaque compromet son utilisation [20].

\section{Aspects techniques}

1.4 Au cours de l'ECMO, le débit obtenu au niveau de la canule de drainage est un déterminant essentiel de l'efficacité de l'oxygénation.

1.5 L'insertion et l'utilisation des canules coaxiales exposent à un risque accru de perforation myocardique. 
1.6 Au cours de l'ECMO, le choix du site d'insertion et des diamètres des canules doit permettre de générer un débit d'assistance adapté aux objectifs thérapeutiques.

1.7 Chez l'adulte, au cours de l'ECMO veinoveineuse, la configuration fémorojugulaire interne doit être favorisée pour permettre de générer un débit d'assistance adapté aux objectifs thérapeutiques (RC).

1.8 Au cours de l'ECMO, l'utilisation de pompes centrifuges non occlusives doit être favorisée (RC).

1.9 Au cours de l'ECMO, l'utilisation d'oxygénateurs à membrane en polyméthylpentène doit être favorisée $(\mathrm{RC})$.

\section{Circuit d'ECMO}

Il comprend les éléments suivants assemblés en série : une canule de drainage, une pompe centrifuge, un oxygénateur assurant oxygénation et élimination du $\mathrm{CO}_{2}$ et une canule de réinjection. Il est nécessaire d'ajouter un mélangeur de gaz et un système de réchauffement du sang. Toute brèche dans le circuit située en amont de la pompe sera responsable d'admission d'air. À l'inverse, toute brèche au-delà de la pompe sera responsable de fuite de sang. Il est déconseillé d'ajouter un accès sur le circuit en amont de la pompe (risque d'admission d'air) [18].

\section{Canules}

La majorité des canules sont en polychlorure de vinyle ou en silicone. Elles sont préhéparinées ou disposent d'un revêtement qui en augmente la biocompatibilité [22]. L'introducteur doit s'adapter très étroitement à la canule, afin de faciliter la pénétration de la canule dans la paroi du vaisseau. Les canules doivent être positionnées en respectant l'axe du membre afin d'éviter les plicatures. Leur fixation doit être assurée par au moins trois points. La qualité de la fixation doit être vérifiée à chaque prise de poste de l'équipe infirmière.

\section{Au cours de l'ECMO VV}

Le débit obtenu au niveau de la canule de drainage est un déterminant essentiel de l'efficacité de l'oxygénation [22]. Le débit maximal attendu avec une canule est proportionnel à son rayon interne élevé à la puissance 4 et inversement proportionnel à sa longueur. Le débit généré doit être au moins de $60 \%$ du débit cardiaque théorique du patient afin d'obtenir une saturation de l'hémoglobine au niveau artériel suffisante [23]. Pour ce faire, le diamètre des canules de drainage doit varier de 24 à $31 \mathrm{~F}$. Les canules doivent être rigidifiées jusqu'à leur extrémité au moyen d'une armature métallique. L'utilisation de canules de drainage multiperforées permet de limiter les lésions de succion endothéliale. Le débit d'assistance est moins dépendant du diamètre de la canule de réinjection. Un diamètre de 16 à $23 \mathrm{~F}$ est généralement suffisant pour prévenir le risque d'hémolyse. Des canules coaxiales ou double-lumières permettent à la fois le drainage et la réinjection. Ce type de canule, insérée en jugulaire interne, permet un drainage veineux cave supérieur et inférieur et une réinjection directement au niveau de la valve tricuspide [18]. Ce type de canule expose à un risque accru de perforation myocardique [24]. Il est indispensable de s'assurer que le guide et la canule ne se situent ni dans les veines sus-hépatiques ni au niveau de la valve tricuspide, mais bien dans la veine cave inférieure.

\section{- En pratique}

Les configurations fémorojugulaire, fémorofémorale, jugulofémorale ou jugulaire (au moyen d'une canule à double lumière) sont possibles. C'est la conformation fémorojugulaire qui permet le meilleur compromis entre débit et oxygénation [22]. L'extrémité de la canule de drainage insérée par voie fémorale doit se situer entre 5 et $10 \mathrm{~cm}$ de la jonction veine cave inférieure-oreillette droite. L'extrémité de la canule de réinjection insérée en jugulaire interne droite doit se situer à la jonction veine cave supérieure-oreillette droite. Cette conformation permet généralement d'atteindre un débit de pompe de 5 à $61 / \mathrm{min}$. En cas d'insertion fémorofémorale, l'extrémité de la canule de drainage se situe entre 5 et $10 \mathrm{~cm}$ de la jonction veine cave inférieure-oreillette droite. L'extrémité de la canule de réinjection insérée en fémoral se situe à la partie basse de l'oreillette droite [22]. La configuration fémorofémorale s'accompagne d'une recirculation importante qui en limite l'intérêt.

\section{Au cours de l'ECMO VA}

La problématique majeure de la canulation artérielle est le risque d'ischémie d'aval liée à l'obstruction de l'artère fémorale commune, qui impose d'implanter en aval un dispositif de reperfusion [22]. L'extrémité de la canule de réinjection insérée au niveau artériel fémoral est placée au niveau de la partie moyenne de l'aorte ascendante. L'extrémité de la canule de drainage doit être placée au niveau de l'oreillette droite.

\section{- En pratique}

L'implantation fémorofémorale est la plus simple et la plus utilisée en urgence. La réinjection peut également se faire en axillaire [19]. Cet abord périphérique fémorofémoral est moins complexe que l'abord central qui nécessite une sternotomie. L'ECMO VA périphérique ne permet cependant pas la décharge du ventricule gauche. Le drainage veineux étant incomplet, il persiste une circulation de l'oreillette droite vers le ventricule gauche à même de générer un débit 
qui entre en compétition avec le sang réinjecté de façon rétrograde par la canule artérielle de réinjection. Il en résulte une perfusion de la partie supérieure du corps (vascularisée par les artères naissant de la crosse de l'aorte) par du sang imparfaitement oxygéné, alors que la partie inférieure du corps reçoit du sang correctement oxygéné provenant du circuit extracorporel (syndrome d'Arlequin). Pour éviter le syndrome d'Arlequin, il faut réduire le débit généré par le ventricule gauche en insérant une canule de décharge supplémentaire (au niveau du ventricule droit, de l'artère pulmonaire ou du ventricule gauche) ou convertir l'ECMO VA périphérique en ECMO VA centrale [22].

\section{Pompes}

On distingue les pompes non occlusives et les pompes occlusives $[25,26]$. Les pompes occlusives sont généralement réservées aux techniques peropératoires de circulation extracorporelle. Les pompes non occlusives sont des pompes rotatives centrifuges, constituées d'un rotor mobile, électrique, qui propulse le sang soit par l'intermédiaire d'ailettes ou de cône (effet vortex), soit par hélice. Elles sont pré- et postcharge dépendantes, et sont actuellement les seules utilisées en Europe. Ces pompes rotatives centrifuges sont entraînées par une force magnétique qui peut générer une vitesse allant jusqu'à 7000 tours/minute. Cette force magnétique a pour fonction de drainer le sang veineux et de produire une force centrifuge à l'origine du débit de pompe continu et non pulsé. Ces pompes rotatives centrifuges permettent le maintien de l'assistance extracorporelle sur une durée prolongée avec un faible risque de stagnation sanguine et de rupture [27]. Le débit de pompe adapté aux objectifs thérapeutiques est fonction de la vitesse de rotation, des pressions d'entrée et de sortie et de la taille des canules [28].

\section{Oxygénateur}

On distingue deux types d'oxygénateurs, les oxygénateurs à bulles utilisés lors de la circulation extracorporelle peropératoire de chirurgie cardiaque et les oxygénateurs à membrane, qui évitent l'hémotraumatisme lié au bullage $[25,26]$. Seuls ces derniers sont utilisés pour l'ECMO périphérique. Il existe deux types de membranes : les membranes planes et les membranes tubulaires [28]. Les oxygénateurs à membranes planes comportant des membranes en silicone ou des membranes assemblées en couches (oxygénateurs à plaque). Les oxygénateurs à membranes tubulaires sont composés de fibres creuses composées pour la plupart de polyméthylpentènes (PMP) non poreuses, évitant la perte des composants du plasma. L'oxygénateur doit être situé en aval de la pompe et permet les échanges gazeux en reproduisant artificiellement la fonction de la membrane alvéolocapillaire. Il assure également les échanges thermiques et le réchauffement du sang du circuit extracorporel [25]. Il comprend une admission et une sortie et est relié à un mélangeur de gaz qui permet le réglage de la fraction en oxygène délivrée par le circuit extracorporel $\left(\mathrm{F}_{\mathrm{ec}} \mathrm{O}_{2}\right)$ et du débit de gaz (débit de balayage). Les oxygénateurs sont testés pour mesurer les pressions en fonction des débits. On admet que le gradient de pression généré par le passage du sang à travers l'oxygénateur doit être inférieur à $50 \mathrm{mmHg} / \mathrm{l}$ par minute. La perte de charge correspond au gradient de pression transmembranaire. Les objectifs thérapeutiques distinguent l'oxygénation et la décarboxylation. Les performances de la membrane sont évaluées par le rated flow de l'oxygénateur qui correspond à la quantité de sang veineux pouvant être oxygéné de 75 à $95 \%$ de saturation en oxygène dans un intervalle de temps donné. Le débit de balayage est réglé au même débit que celui du débit de l'assistance. La majorité des oxygénateurs supportent des débits de balayage de $10 \mathrm{ml} / \mathrm{min}$ à $100 \mathrm{l} / \mathrm{min}$ [25]. L'oxygénateur doit être placé sous le niveau du patient. Le port de sortie des gaz doit être de préférence dirigé vers le bas afin de limiter les risques d'obstruction [25].

\section{Particularités pédiatriques}

Au cours de l'ECMO VV chez l'enfant de moins de $15 \mathrm{~kg}$, il est recommandé de drainer à partir de la veine cave supérieure, de façon à obtenir un meilleur débit de pompe, car un drainage par voie fémorale avec une canule longue mais fine et résistive en position intra-auriculaire droite entraîne une perte de charge délétère au fonctionnement de la circulation extracorporelle [29,30]. Au cours de l'ECMO VA chez l'enfant de moins de $10 \mathrm{~kg}$, il est recommandé de réaliser les canulations au niveau cervical [25]. À partir de 30 à $40 \mathrm{~kg}$, les techniques sont comparables à celles de l'adulte.

En cas d'utilisation de canule unique à simple lumière (de 10 à 14 Fr.), l'introduction se fait par voie jugulaire interne droite jusque dans l'oreillette droite. L'alternance drainage et réinjection par la même canule est permise grâce à l'utilisation d'une pompe non occlusive et d'un clamp alternatif [31]. Les débits utilisés sont un peu plus élevés (environ $30 \%$ du débit cardiaque). La recirculation est faible.

\section{Quels patients relèvent d'une assistance extracorporelle au cours du SDRA ?}

\section{Principes généraux}

\subsection{Les indications de l'ECMO doivent reposer sur une décision collégiale et multidisciplinaire, rensei- gnée dans le dossier médical (RC).}


2.2 Au cours du SDRA, il faut discuter les indications d'ECMO au cas par cas, en prenant en compte le rapport bénéfice/risque.

2.3 L'information et la recherche du consentement du patient, à défaut de la personne de confiance, sont des préalables obligatoires à la mise en œuvre de l'ECMO au cours du SDRA.

2.4 Lorsque le patient est hors d'état d'exprimer sa volonté, et en dehors des situations d'urgence, l'information des proches est un préalable obligatoire à la mise en ouvre de l'ECMO au cours du SDRA.

2.5 La réversibilité prévisible des lésions pulmonaires et l'absence de toute autre limitation thérapeutique constituent un prérequis indispensable à l'utilisation de l'ECMO (RC).

2.6 Devant une forme sévère de SDRA, il ne faut pas recourir à l'ECMO tant qu'une stratégie de ventilation protectrice comportant, lorsqu'il est possible, le recours au décubitus ventral n'a pas été mise en place.

2.7 Chez l'enfant, lorsque la $\mathrm{PaO}_{2}$ n'est pas disponible, il est possible d'évaluer la gravité du SDRA en utilisant le ratio $\mathrm{SpO}_{2} / \mathrm{FiO}_{2}(\mathrm{RC})$.

Le SDRA est une maladie pulmonaire inflammatoire évolutive avec une distribution hétérogène de l'atteinte de la membrane alvéolocapillaire. Ces lésions sont responsables d'anomalies progressives de l'hématose à l'origine de la détresse respiratoire [10]. En plus du traitement étiologique, le recours à une assistance respiratoire est nécessaire dans les formes graves. Cependant, il est démontré que la ventilation mécanique est par elle-même à l'origine d'une aggravation des lésions pulmonaires connues sous le terme de VILI (ventilator-induced lung injury) [2]. Des études expérimentales et des essais cliniques ont montré que ces lésions peuvent être réduites en optimisant les réglages du ventilateur [2]. En conséquence, devant une forme sévère de SDRA, il n'est pas raisonnable de recourir à une technique d'assistance extracorporelle tant qu'une stratégie de ventilation protectrice n'a pas été mise en place. Celle-ci repose sur [1] :

- un volume courant entre 4 et $8 \mathrm{ml} / \mathrm{kg}$ de poids théorique prédit par la taille ;

- une pression expiratoire positive (PEP) élevée ;

- une pression de plateau inférieure ou égale à $30 \mathrm{cmH}_{2} \mathrm{O}$;

- une curarisation au cours des 48 premières heures ;

- une épreuve de décubitus ventral [32].

Chez l'enfant de moins de $20 \mathrm{~kg}$, l'optimisation de la ventilation mécanique peut nécessiter l'utilisation de la ventilation en oscillation à haute fréquence [33].

Compte tenu du niveau de preuve actuel des études cliniques sur l'utilisation de l'ECMO au cours du SDRA, de l'impact médico-économique de la diffusion de ces techniques et de la charge en soins considérable qui l'accompagne, les indications doivent être discutées au cas par cas en fonction du rapport bénéfice/risque individuel. L'information et le consentement du patient, à défaut de la personne de confiance, sont des préalables obligatoires à la mise en œuvre de ces techniques. Chez l'enfant, l'autorisation de la personne exerçant l'autorité parentale est nécessaire en regard du texte législatif (article R.1112-35 du Code de santé publique) concernant tout acte chirurgical. Enfin, la réversibilité prévisible des lésions pulmonaires et l'absence de toute décision de limitation thérapeutique sont des prérequis indispensables.

Techniques d'assistance extracorporelle recommandées au cours du SDRA

2.8 Parmi les techniques d'assistance extracorporelle, l'ECMO veinoveineuse est la technique de référence au cours du SDRA sévère.

2.9 Il ne faut pas, en l'état actuel des connaissances, utiliser les techniques d'épuration du $\mathrm{CO}_{2}$ à faible débit $\left(\mathrm{ECCO}_{2}-\mathrm{R}\right)$ au cours du SDRA.

2.10 Les techniques d'épuration du $\mathrm{CO}_{2}$ à faible débit $\left(\mathrm{ECCO}_{2}-\mathrm{R}\right)$ doivent faire l'objet d'une évaluation dans le cadre d'essais cliniques (RC).

Les résultats négatifs des deux études multicentriques prospectives randomisées publiées en 1979 et 1994 [4,34] à propos de l'utilisation de l'ECMO dans la prise en charge du SDRA sévère $\left(\mathrm{PaO}_{2} / \mathrm{FiO}_{2}<50 \mathrm{mmHg}\right)$ avaient conduit à l'abandon de cette technique. Les raisons de l'échec de la technique rapportées dans ces études étaient multiples : utilisation tardive de l'ECMO après une période de ventilation mécanique prolongée associée à une mortalité d'environ $90 \%$, utilisation de l'ECMO VA avec le risque d'hypoxie cérébrale secondaire au syndrome d'Arlequin, grande fréquence des complications hémorragiques cérébrales et utilisation de modalités de ventilation non protectrice à l'origine d'une incidence majeure de complications baro- et volotraumatique pulmonaire [4,34].

Prenant en compte le concept de la mise au repos du poumon et celui de la nécessité de limiter au maximum la pression intra-alvéolaire lors de la ventilation mécanique en pression positive, de nombreuses études observationnelles ont rapporté, en particulier à l'initiative de Gattinoni et al. [3], des survies de l'ordre de $50 \%$ [9].

Plus récemment, l'épidémie de grippe H1N1 de 2009 a conduit, compte tenu de l'existence de formes très sévères de SDRA survenant chez des sujets jeunes, de l'amélioration des matériaux disponibles (canules, pompes et membranes) et d'une connaissance plus précise des modalités de la ventilation protectrice, à un regain d'intérêt de l'utilisation de l'ECMO. L'analyse des études observationnelles [5-8] suggère un effet bénéfique en termes de survie en particulier pour les patients les plus graves même si d'autres études observationnelles rapportées par des équipes n'utilisant pas l'ECMO semblent avoir rapporté des effets comparables [35]. 
La seule étude prospective randomisée associant l'utilisation de l'ECMO à une ventilation protectrice a été réalisée par Peek et al. chez 180 patients souffrant de SDRA sévère (score de Murray $>3$ ou $\mathrm{pH}<7,20$ ). Elle a mis en évidence une réduction significative d'un critère composite associant la mortalité et le handicap sévère à six mois ( 37 vs $53 \%$ ) chez les patients recevant l'ECMO [7]. Même si le protocole de cette étude, qui comportait le transfert vers un centre référent des patients inclus dans le bras ECMO et le recours systématique à une ventilation protectrice uniquement dans ce même bras, ne permet pas la comparaison de l'ECMO avec la prise en charge optimisée du SDRA, il met en exergue l'intérêt du recours à un centre compétent dans l'utilisation non seulement de la technique d'ECMO, mais également du traitement du SDRA sévère.

Cette publication a également précisé l'intérêt d'une équipe mobile permettant le transfert des malades instables et sévèrement hypoxiques et a posé la question de l'impact de l'effet volume au sein d'équipes formées et entraînées sur les résultats associés à l'utilisation de l'ECMO [7]. En dépit du caractère insuffisant du niveau de preuve fourni par cette analyse de la littérature, le jury considère qu'il existe donc des indications consensuelles à l'utilisation de l'ECMO VV.

Par contre, en l'état actuel des données cliniques disponibles, il n'est pas recommandé d'utiliser l'ECCO $-\mathrm{R}$ dans le SDRA. Cependant, cette approche pourrait faciliter la mise en œuvre de stratégies de ventilation plus protectrices que celles préconisées aujourd'hui afin de limiter davantage le traumatisme pulmonaire au cours de la ventilation mécanique : par exemple par l'utilisation de volumes courants inférieurs à $4 \mathrm{ml} /$ $\mathrm{kg}$ de poids prédit par la taille et de pressions de plateau inférieures à $25 \mathrm{cmH}_{2} \mathrm{O}$. Ces indications devront faire l'objet d'une évaluation dans le cadre d'essais cliniques prospectifs [12].

\section{Indications de l'ECMO}

2.11 Il faut envisager le recours à l'ECMO VV si le rapport $\mathrm{PaO}_{2} / \mathrm{FiO}_{2}$ est inférieur à $50 \mathrm{mmHg}$ sous une $\mathrm{FiO}_{2}=1$, pendant au moins trois heures en dépit d'une stratégie de ventilation protectrice (comportant le recours au décubitus ventral) [RC].

2.12 Il faut engager une réflexion sur le recours à l'ECMO $\mathrm{VV}$ si le rapport $\mathrm{PaO}_{2} / \mathrm{FiO}_{2}$ est inférieur à $80 \mathrm{mmHg}$ sous une $\mathrm{FiO}_{2}=1$, pendant plus de six heures en dépit d'une stratégie de ventilation protectrice (comportant le recours au décubitus ventral) [RC].

2.13 Il faut engager une réflexion sur le recours à l'ECMO VV si, associée à une stratégie de ventilation protectrice (comportant le recours au décubitus ventral), existe une acidose respiratoire avec un $\mathrm{pH}$ inférieur à 7,20 pendant plus de six heures (RC).

2.14 Il n'existe pas d'indication de l'ECMO VA au cours du SDRA lorsque la défaillance respiratoire est isolée. Un choc cardiogénique associé peut la faire discuter (RC).
2.15 Lorsqu'elle fait décider de l'ECMO, la présence d'un cour pulmonaire aigu au cours du SDRA n'est pas une indication obligatoire à l'ECMO VA (RC).

En dépit du caractère insuffisant du niveau de preuve fourni par l'analyse de la littérature, le jury de la conférence de consensus considère qu'il faut cependant envisager le recours à l'ECMO VV si l'hypoxémie se traduit par un rapport $\mathrm{PaO}_{2} / \mathrm{FiO}_{2}$ inférieur à $50 \mathrm{mmHg}$ (mesuré sous une $\mathrm{FiO}_{2}=1$ ) pendant plus de trois heures. Compte tenu de l'évolutivité prévisible de l'atteinte pulmonaire et des délais nécessaires à la mise en œuvre de la technique, il considère comme raisonnable d'engager, de manière anticipée, une réflexion collégiale sur l'éventualité de ce recours lorsque persistent pendant plus de six heures une hypoxémie avec un rapport $\mathrm{PaO}_{2} / \mathrm{FiO}_{2}$ inférieur à $80 \mathrm{mmHg}$ et/ou une acidose respiratoire avec un pH inférieur à 7,20. Les indications d'ECMO VA sont extrêmement limitées dans le SDRA. Elles peuvent cependant se discuter en présence d'un choc cardiogénique associé [36].

\section{Contre-indications}

2.16 L'impossibilité de recourir à une anticoagulation est une contre-indication classique à l'ECMO (RC).

2.17 Le rapport bénéfice/risque de l'ECMO au cours du SDRA doit être considéré comme défavorable en cas de : 1) lésions intracrâniennes hémorragiques ou potentiellement hémorragiques ; 2) coma dans les suites d'un arrêt cardiaque ; 3) SDRA dont la durée de ventilation mécanique excède sept jours ; 4) immunodépression sévère ; 5) syndrome de défaillance multiviscérale (SOFA > 15) $(\mathrm{RC})$.

La seule contre-indication absolue à l'ECMO est représentée par l'impossibilité de recourir à une anticoagulation [9]. Même si la littérature ne fournit pas d'argument définitif en faveur de contre-indications relatives, il apparaît dans les expériences rapportées que certains sous-groupes de patients sont susceptibles de moins bénéficier de ces techniques. Lors de la discussion collégiale de l'indication d'assistance extracorporelle, il faudra donc tenir compte de la survenue du SDRA dans les situations suivantes [28] : hémorragie cérébrale, coma postarrêt cardiaque lorsque l'état neurologique ne peut être apprécié objectivement, durée de ventilation mécanique supérieure à sept jours, immunodépression sévère, défaillance multiviscérale évaluée par un score SOFA supérieur à 15 .

\section{Comment réaliser l'assistance extracorporelle au cours du SDRA ?}

\section{Principes généraux}

3.1 Le montage, la pose et la gestion quotidienne de l'ECMO doivent être formalisés. Dans ce but, l'utilisation de « check-lists » est recommandée (RC). 
3.2 Pour la mise en place et la gestion d'une ECMO, la présence d'un personnel médicosoignant formé au montage du circuit est recommandée ( $\mathrm{RC})$.

3.3 Au cours de l'ECMO, un réglage de la ventilation mécanique permettant de limiter au maximum le niveau de pression de plateau tout en administrant un niveau minimum de PEP est nécessaire.

L'ECMO reste une procédure d'exception, qui doit être encadrée par une surveillance formalisée. L'utilisation de " check-lists », qui a montré son efficacité au bloc opératoire en général et lors de procédures de circulation extracorporelle, doit faire partie de cette surveillance [37]. Parmi les éléments majeurs de la surveillance figure un relevé régulier des vitesses de rotation de la pompe et des débits obtenus. La batterie de la pompe ne permettant qu'une heure d'autonomie, le branchement électrique doit donc être régulièrement vérifié. La pompe et le circuit sont munis d'alarmes qui permettent de signaler une inversion de débit. La mise à disposition d'une manivelle, d'une tête de secours manuelle permettant de remplacer en urgence la pompe en cas de dysfonctionnement et de clamps doit être quotidiennement vérifiée. La survenue de complications (saignements, infection de canules, embolies, hémolyse) doit également être systématiquement recherchée.

Cette surveillance spécifique requiert du personnel formé, capable de réagir en urgence devant une complication, et à même de remonter un circuit d'ECMO à tout moment. À titre d'exemple, le débullage d'un circuit d'ECMO étant une étape cruciale associée au risque d'embolie gazeuse, ce personnel doit y avoir été parfaitement formé. Ainsi, ce personnel devrait comporter dans le meilleur des cas un perfusionniste habitué à la gestion des circuits. Ce dernier participera à la formation du personnel médical et soignant.

Sous ECMO, quel que soit le mode de ventilation choisi, il faut utiliser un mode de ventilation " protecteur ». Les données récentes montrent que la survie des patients sous ECMO est meilleure lorsque la pression de plateau est en médiane autour de $25 \mathrm{cmH}_{2} \mathrm{O}$ [8]. La fréquence respiratoire pourra être diminuée jusqu'à moins de dix cycles par minute tandis que la $\mathrm{FiO}_{2}$ sera réduite progressivement en dessous de 0,6 (en même temps que la $\mathrm{F}_{\mathrm{ec}} \mathrm{O}_{2}$ ) [8]. Au cours de l'ECMO, l'utilisation d'une ventilation dite « ultraprotectrice » avec des volumes inférieurs à $3 \mathrm{ml} / \mathrm{kg}$ doit être évaluée en termes de pronostic [12].

\section{Canulation}

3.4 Lors de la canulation percutanée, la possibilité de recourir en urgence à une compétence chirurgicale vasculaire et thoracique doit être organisée (RC).

3.5 Pour l'insertion percutanée des canules d'ECMO, il faut réaliser la ponction vasculaire par échoguidage.
3.6 Le bon positionnement des canules d'ECMO doit être contrôlé par échographie et radiographie de thorax (RC).

La pose d'une ECMO VV, réalisée en condition d'asepsie chirurgicale, justifie la présence de deux opérateurs. En l'absence de chirurgien cardiaque lors de la canulation percutanée, la possibilité d'avoir recours à une compétence chirurgicale vasculaire et thoracique en urgence en cas de complication est une nécessité [18]. En effet, la survenue de complications parfois létales (hémorragies, fausses routes, dissection, perforations) lors de la canulation est décrite dans près de $10 \%$ des cas. En regard des recommandations existantes pour la ponction vasculaire en réanimation, un échoguidage est recommandé.

Pour l'ECMO VA, la canulation fémorofémorale par voie chirurgicale est la voie de référence. Elle nécessite une compétence chirurgicale. Les complications liées à l'ECMO VA sont nombreuses, parfois fatales dans un délai extrêmement bref, ce qui justifie une prise en charge par des équipes médicochirurgicales habituées aux techniques d'implantation, mais également à la gestion de leurs complications.

L'utilisation de l'échographie lors de la mise en place et de la réalisation de l'ECMO est indispensable [38,39]. La progression des guides ainsi que la bonne position des canules pourront être surveillées par échographie transthoracique au lit du patient lors de leur implantation permettant ainsi de limiter le phénomène de recirculation lié à leur mauvaise position. Le positionnement des canules coaxiales est particulièrement délicat. Plusieurs techniques sont proposées pour améliorer la mise en place et prévenir les complications liées à leur pose $[24,40,41]$. L'échographie permet enfin d'évaluer la fonction myocardique et de déterminer l'existence éventuelle d'une précharge dépendance.

\section{Réglages de l'ECMO}

3.7 Pour une efficacité optimale de l'oxygénation par ECMO VV, le débit de la pompe à sang doit être supérieur ou égal à $60 \%$ du débit cardiaque théorique.

3.8 La fraction en oxygène délivrée par le circuit extracorporel $\left(\mathrm{F}_{\mathrm{ec}} \mathrm{O}_{2}\right)$ doit permettre d'obtenir une $\mathrm{SaO}_{2}$ supérieure ou égale à $88 \%$.

3.9 Le débit de balayage doit permettre d'obtenir une $\mathrm{PaCO}_{2}$ comprise entre 30 et $40 \mathrm{mmHg}$.

Les réglages sont effectués au moyen d'une console de contrôle. La vitesse de rotation de la pompe est réglée en tours/minute. Elle doit être supérieure à 1500 tours/minute. Les pompes centrifuges peuvent générer une vitesse allant jusqu'à 7000 tours/minute. La vitesse idéale se situe entre 3000 et 3500 tours/minute. Le débit de pompe mesuré par un capteur à ultrasons doit être supérieur à $2 \mathrm{l} / \mathrm{min}$. Au niveau de l'oxygénateur, le débit de balayage qui conditionne le 
niveau de la $\mathrm{PaCO}_{2}$ du patient doit être d'une à deux fois celui du débit de la pompe [25].

\section{Anticoagulation du circuit extracorporel}

3.10 Une anticoagulation par héparine non fractionnée doit être instituée avec pour objectif un temps de céphaline activé (TCA) entre 1,2 et 1,5 $\times$ témoin (ou une activité anti-Xa entre 0,2 et $0,4 \mathrm{UI} / \mathrm{ml}$ ) [RC].

3.11 En cas de saignement significatif, l'anticoagulation doit être diminuée ou totalement arrêtée. La poursuite de l'ECMO doit être discutée (RC).

3.12 Chez l'enfant, un monitorage précis de l'hémostase est nécessaire comportant notamment le thromboélastogramme et la mesure de l'activité anti-Xa (RC).

Les principales complications de l'ECMO sont d'ordre hémorragique. Leur incidence paraît proche de $50 \%$ [42]. Celle des hémorragies intracrâniennes atteint 10-12\% dans les séries où les niveaux d'anticoagulation utilisés étaient plus importants (objectif de TCA autour de $2 \times$ témoin) et autour de $2-4 \%$ dans la série REVA, avec des objectifs d'anticoagulation plus faibles [8]. L'incidence de la coagulation de circuit est peu décrite, probablement de l'ordre de 15 à $20 \%$. Elle semble nettement diminuée avec l'utilisation des circuits imprégnés d'anticoagulants.

La balance bénéfice/risque est en faveur de l'utilisation des niveaux d'anticoagulation les plus bas associés à des vitesses élevées de rotation de la pompe. Il est d'usage de réaliser un bolus d'héparine lors de la canulation. La surveillance de la coagulation à l'aide d'un thromboélastogramme a été proposée en particulier en pédiatrie.

L'ECMO, comme toute circulation extracorporelle, peut favoriser la production d'anticorps anti-PF4 et donc la survenue de thrombopénie immunoallergique à l'héparine [43]. Même si les doses reçues d'héparine sont moindres sous ECMO, la recherche de ce diagnostic devant une thrombopénie inexpliquée est licite. Même si la fréquence du déficit en antithrombine III, à l'origine d'une résistance à l'héparine est faible, il est recommandé de réaliser ce dosage compte tenu de la gravité des conséquences d'une coagulation du circuit et de l'intérêt reconnu d'une substitution pour restaurer l'efficacité de l'héparine.

Causes de dysfonctionnement de la circulation extracorporelle

3.13 Au cours de l'ECMO, lorsque le débit de la pompe à sang chute malgré le maintien de la vitesse de rotation, une réduction de précharge ou une augmentation de postcharge doivent être recherchées.

3.14 Lors de la mise en route d'une ECMO VV pour SDRA, l'aggravation ou la persistance d'une hypoxémie peut être secondaire à la levée de la vasoconstriction pulmonaire hypoxique, mais doit faire rechercher une cause mécanique (défaillance de l'oxygénateur, débit insuffisant, recirculation) [RC].

3.15 Lors de la réalisation d'une ECMO VV pour SDRA, l'aggravation d'une hypoxémie peut être secondaire à une aggravation pulmonaire, mais doit faire rechercher une cause mécanique (défaillance de l'oxygénateur, débit insuffisant, recirculation) [RC].

Les causes principales de réduction de précharge sont l'hypovolémie ou un dysfonctionnement de la canule de drainage (thrombose ou mobilisation). La cause principale d'augmentation de postcharge est un dysfonctionnement de la canule de réinjection (thrombose, mobilisation). L'hypovolémie est également identifiable par la constatation d'un tremblement des lignes de l'ECMO. Un débit trop élevé peut entraîner des phénomènes de succion au niveau des zones de drainage veineux à l'origine de l'altération de l'endothélium vasculaire. Le risque est alors celui de la chute du débit de la pompe en raison du collapsus du système d'assistance.

Les principales causes d'hypoxémie persistante sont rappelées dans le Tableau 2. Dans ces situations, il convient de se rappeler que le transport en oxygène dépend également du débit cardiaque et du taux d'hémoglobine, paramètres sur lesquels il est possible d'agir.

Tableau 2 Causes d'hypoxémie sous ECMO et actions proposées

Causes possibles Actions proposées

Recirculation importante

Débit insuffisant par rapport à la demande métabolique

Défaillance de l'oxygénateur

Majoration du shunt pulmonaire secondaire à la levée de la vasoconstriction pulmonaire hypoxique

Aggravation de la fonction pulmonaire (pneumonie, pneumothorax...)

\section{Vérifier la position des canules}

Adapter le diamètre des canules et optimiser l'état volémique Évaluer le fonctionnement de l'oxygénateur en mesurant la $\mathrm{PO}_{2}$ efférente

Évaluer le shunt en mesurant les $\mathrm{PO}_{2}$ du circuit et du patient 


\section{Particularités pédiatriques}

Chez l'enfant de moins de $10 \mathrm{~kg}$ (volume sanguin de $80 \mathrm{ml} / \mathrm{kg}$ ), le priming par du sérum physiologique d'un circuit dont le volume excède $200 \mathrm{ml}$ peut être responsable d'une hémodilution. Il est donc nécessaire d'assurer le remplissage du circuit soit avec du sang, soit avec du plasma frais, soit avec de l'albumine [44]. Une hémofiltration couplée à l'ECMO permet d'équilibrer la balance hydrosodée et les apports caloriques [45]. La surveillance de l'oxygénation tissulaire cérébrale à l'aide de la spectroscopie proche de l'infrarouge a été proposée [46]. Une fréquence plus élevée que chez l'adulte d'accidents vasculaires cérébraux est rapportée chez l'enfant. Ces accidents vasculaires cérébraux peuvent être ischémique $(4 \%)$ ou hémorragique $(6 \%)$ [42]. L'ECMO VA est associée à plus de complications neurologiques cérébrales que l'ECMO VV [47,48]. Dans ce contexte, une surveillance de l'anticoagulation à l'aide du thromboélastogramme et du dosage de l'anti-Xa est souhaitable. Enfin, la mise en décubitus ventral améliore le pronostic des enfants placés sous ECMO [49].

\section{Quand et comment arrêter une circulation extracorporelle au cours du SDRA ?}

\section{Principes généraux}

\subsection{La question du sevrage de l'ECMO doit être posée} de façon quotidienne (RC).

4.2 Concernant la poursuite de l'ECMO, la question de l'obstination déraisonnable doit être discutée au cours de réunions collégiales et multidisciplinaires (RC).

4.3 L'arrêt de l'ECMO est fortement recommandé lors de la survenue d'une complication cérébrale grave hémorragique ou embolique (RC).

4.4 Chez l'enfant, les modalités de sevrage de l'ECMO ne sont pas différentes de celles de l'adulte (RC).

L'ECMO est une technique lourde, consommatrice de soins et associée à des complications potentiellement graves. Sa durée d'utilisation doit être la plus courte possible, et la question de la récupération de la fonction pulmonaire et donc de l'arrêt de l'ECMO doit être posée quotidiennement.

Une décision d'arrêt de l'ECMO peut être prise lorsque sa poursuite paraît relever de l'obstination déraisonnable. Dans ce contexte, aucun critère précis d'arrêt de l'ECMO n'a cependant été déterminé. Il a, d'autre part, été rapporté que la guérison et la récupération de la fonction pulmonaire pouvaient nécessiter plusieurs semaines $[9,22,50]$. Certains critères cliniques, observés dès les premiers jours d'un traitement par ECMO, ont été associés à un mauvais pronostic tels que l'absence d'amélioration de la pression de plateau, de la lactatémie ou de la défaillance hémodynamique $[8,23,52,57]$. La durée moyenne de maintien de l'ECMO étant dans la littérature d'une à deux semaines, la question de l'arrêt de celle-ci doit être posée au-delà de ce délai sous la forme d'une discussion collégiale dans laquelle seront pris en compte, comme dans toute démarche de limitation, des thérapeutiques actives : la réversibilité prévisible de la défaillance pulmonaire et/ou cardiaque et des autres défaillances d'organe, le terrain et dans des circonstances exceptionnelles l'existence d'options thérapeutiques alternatives comme par exemple la transplantation pulmonaire. Si la décision d'arrêt de l'ECMO est prise, elle devra être expliquée aux proches.

\section{Aspects pratiques du sevrage de l'ECMO}

4.5 La procédure de sevrage de l'ECMO comporte la recherche quotidienne de critères témoignant d'une récupération de la défaillance respiratoire ou cardiorespiratoire (RC).

4.6 Lors de la procédure de sevrage de l'ECMO, l'absence de cour pulmonaire aigu doit être recherchée (RC).

4.7 L'arrêt de l'ECMO est décidé selon les résultats d'une procédure de sevrage formalisée de plusieurs heures (RC).

4.8 Le sevrage de l'ECMO VA impose le maintien d'un balayage sur l'oxygénateur (RC).

4.9 Au cours du SDRA, l'absence de sevrage possible d'une ECMO VA doit faire discuter la possibilité d'une conversion en ECMO VV (RC).

Le sevrage de l'ECMO VV est réalisé avant celui de la ventilation mécanique. Il serait souhaitable d'évaluer l'intérêt d'une stratégie d'extubation sous ECMO pour permettre la mobilisation plus précoce des patients.

Il est recommandé, pendant la procédure de sevrage de l'ECMO, de mesurer les conséquences des modifications du débit de balayage sur la capnie dont les variations peuvent être délétères en particulier au niveau de la circulation cérébrale. En cas d'hypercapnie persistante, le recours aux méthodes d'épuration du $\mathrm{CO}_{2}$ à faible débit pourrait être envisagé, mais l'intérêt de cette option n'a pas été évalué.

Il n'y a pas de données disponibles justifiant l'utilisation des corticoïdes en cas d'échec du sevrage de l'ECMO.

La constatation d'une récupération de la défaillance respiratoire ou cardiorespiratoire, jugée sur l'amélioration clinique (notamment de la compliance du système respiratoire), gazométrique et radiologique, est un préalable indispensable avant d'envisager une procédure de sevrage de l'ECMO [7,16,28].

À partir des données de la littérature [22,51,52], l'arrêt de l'ECMO VV peut être envisagé devant les résultats d'une procédure de sevrage consistant en une réduction : 
- du balayage de la membrane jusqu'à son arrêt complet ;

- de la $\mathrm{F}_{\text {ec }} \mathrm{O}_{2}$ aux alentours de $21 \%$.

Une réduction du débit de la pompe à 1 à $21 /$ min expose aux risques de coagulation du circuit, et n'est donc pas, pour de nombreux experts, systématiquement nécessaire pour envisager la procédure de sevrage.

En cas d'épreuve de sevrage prolongée, il est recommandé de balayer la membrane de l'oxygénateur par un mélange gazeux à fort débit pendant 30 secondes toutes les heures.

L'arrêt de l'ECMO VV est décidé lorsque l'hématose et la pression de plateau sont jugées satisfaisantes (Tableau 3) $[18,28,51,53]$.

Le sevrage de l'ECMO VA est moins bien codifié, mais des critères voisins de ceux utilisés pour le sevrage de l'ECMO VV sont proposés (Tableau 3). À l'inverse de l'ECMO VV, il faut maintenir un balayage sur l'oxygénateur afin d'éviter de réinjecter du sang veineux dans le système artériel périphérique. Un débit résiduel de 1 1/min est en général utilisé. Si le patient ne peut pas être sevré de l'ECMO VA, sa conversion en ECMO VV doit être discutée, l'ECMO VA étant grevée de plus de complications que l'ECMO VV.

Quel que soit le type d'ECMO, la survenue d'une dysfonction ventriculaire droite lors de la procédure de sevrage devra être évaluée. En cas d'échec du sevrage de l'ECMO VV secondaire à la survenue d'une insuffisance cardiaque droite, un passage à l'ECMO VA pourra être envisagé.

\section{Décanulation}

4.10 L'anticoagulation doit être arrêtée au moins une heure avant la décanulation (RC).

4.11 La décanulation peut être réalisée au bloc opératoire ou en réanimation $(\mathrm{RC})$.
4.12 La décanulation artérielle est toujours un geste chirurgical. La décanulation veineuse peut être médicale ou chirurgicale (RC).

4.13 Chez l'enfant, la décanulation veineuse ou artérielle est toujours chirurgicale $(\mathrm{RC})$.

\section{Quelle organisation proposer?}

Il est nécessaire de proposer une organisation à l'échelon national pour permettre, au cours du SDRA sévère, le recours à l'assistance extracorporelle, en particulier à l'ECMO. Cette organisation doit avoir au minimum les quatre objectifs suivants :

- offrir, à l'ensemble des patients qui le nécessitent, l'accès à l'ECMO dans des conditions de sécurité maximale ;

- répartir les moyens disponibles pour permettre une couverture optimale du territoire ;

- être capable de faire face à une augmentation importante et rapide du nombre de patients à traiter en période épidémique ;

- prendre en compte l'importance des rapports bénéfice/ risque et coût/bénéfice de l'ECMO.

Les données épidémiologiques nationales concernant l'incidence, la sévérité et le pronostic du SDRA en France sont insuffisantes pour estimer de façon fiable le nombre de patients qui relèvent d'un traitement par ECMO. Selon des avis d'experts, le nombre de patients avec SDRA sévère relevant d'un traitement par ECMO se situerait entre cinq à dix par million d'habitants et par an, hors phase épidémique. Une enquête de pratique menée par la SRLF et la Sfar en 2012 auprès de 116 services de réanimation fait état d'environ 300 patients traités par ECMO pour SDRA dans

\begin{tabular}{|c|c|c|}
\hline & Épreuve de sevrage & Critères autorisant l'arrêt de l'ECMO \\
\hline $\begin{array}{l}\text { ECMO } \\
\text { veinoveineuse }\end{array}$ & $\begin{array}{l}\mathrm{F}_{\mathrm{ec}} \mathrm{O}_{2}=21 \% \\
\text { Débit gaz } 11 / \mathrm{min} \text { ou arrêt } \\
\text { Durée du sevrage : plusieurs heures }\end{array}$ & $\begin{array}{l}\text { Pplat }<25-30 \mathrm{cmH}_{2} \mathrm{O} \text { pour VT autour de } 6 \mathrm{ml} / \mathrm{kg} \text { et } \mathrm{PEP}<12 \mathrm{cmH}_{2} \mathrm{O} \\
\text { Et } \mathrm{PaO}_{2}>70 \mathrm{mmHg} \text { sous } \mathrm{FiO}_{2}<60 \% \text { ou } \mathrm{PaO}_{2} / \mathrm{FiO}_{2}>200 \mathrm{mmHg} \\
\text { Et } \mathrm{pH}>7,3 \text { avec } \mathrm{PCO}_{2}<50 \mathrm{mmHg} \\
\text { Et absence de cœur pulmonaire aigu }\end{array}$ \\
\hline $\begin{array}{l}\text { ECMO } \\
\text { veinoartérielle }\end{array}$ & $\begin{array}{l}\mathrm{F}_{\mathrm{ec}} \mathrm{O}_{2}: 21 \% \\
\text { Maintenir débit de gaz à } 11 / \mathrm{min} \\
\text { Diminution débit sang par palier de } 0,5 \mathrm{l} / \mathrm{min} \\
\text { Durée du sevrage : plusieurs heures }\end{array}$ & $\begin{array}{l}\text { Pplat }<25-30 \mathrm{cmH}_{2} \mathrm{O} \text { pour VT autour de } 6 \mathrm{ml} / \mathrm{kg} \text { et } \mathrm{PEP}<12 \mathrm{cmH}_{2} \mathrm{O} \\
\mathrm{Et}^{\mathrm{PaO}_{2}}>70 \mathrm{mmHg} \text { sous } \mathrm{FiO}_{2}<60 \% \text { ou } \mathrm{PaO}_{2} / \mathrm{FiO}_{2}>200 \mathrm{mmHg} \\
\text { Et } \mathrm{pH}>7,3 \text { avec } \mathrm{PCO}_{2}<50 \mathrm{mmHg} \\
\text { Et absence de cœur pulmonaire aigu } \\
\text { En cas de défaillance ventriculaire gauche associée à : } \\
\quad \text { Fraction d'éjection du ventricule gauche }>25-30 \% \\
\quad \text { Intégrale temps-vitesse sous-aortique }>12 \mathrm{~cm}\end{array}$ \\
\hline
\end{tabular}

Pplat : pression de plateau ; VT : volume courant ; PEP : pression expiratoire positive ; $\mathrm{F}_{\mathrm{ec}} \mathrm{O}_{2}$ : fraction en $\mathrm{O}_{2}$ délivrée par le circuit extracorporel 
58 services, confortant l'estimation faite par les experts. Enfin, une requête a été adressée par la SRLF auprès de la base nationale du PMSI. Bien que d'interprétation délicate, elle fait état en 2012 de 679 séjours en réanimation de patients comportant le diagnostic de SDRA et l'utilisation d'une technique d'ECMO VV ou VA, soit un total de 5325 jours de procédure.

L'évolution des techniques et des matériels suggère que l'ECMO VV peut être pratiquée en toute sécurité par la plupart des services de réanimation déjà habitués à la gestion d'autres modes de circulation extracorporelle comme la dialyse et l'hémofiltration continue sous réserve d'une formation spécifique régulière. Cependant, en raison de la gravité potentielle des complications liées à l'implantation d'une ECMO et en raison des compétences nécessaires à la conduite d'un tel traitement, les experts préconisent que les patients relevant de l'ECMO VV ou VA soient regroupés dans des structures disposant de tous les moyens nécessaires à l'indication, à la mise en place et à la conduite du traitement par ECMO en toute sécurité. Dans ces conditions, les structures prenant en charge à la fois les patients atteints de SDRA sévère traités par ECMO VV, et les patients, plus nombreux, nécessitant l'ECMO VA pour des raisons cardiaques, semblent être les structures les plus adaptées. Cela suppose qu'elles disposent au minimum d'un service de réanimation, d'un service de chirurgie cardiaque, ainsi que d'une unité mobile d'assistance circulatoire (UMAC). À titre d'exemple, ce concept de concentration des moyens a été retenu par des pays comme l'Angleterre ou l'Australie, mais pas par d'autres comme l'Allemagne et le Japon. Les preuves apportées par la littérature concernant les avantages d'une telle organisation tant sur le devenir des patients que sur le rapport coût/efficacité sont cependant indirectes et insuffisantes. Comme cela est suggéré en particulier par les études pédiatriques les plus récentes, le nombre de patients traités par ECMO (effet volume associé à cette organisation centralisée) est certainement un élément déterminant pour l'acquisition de l'expertise nécessaire [54,55].

La plupart des expériences rapportées à propos des UMAC plaident en faveur de leur rôle déterminant [56-58]. Cependant, les UMAC françaises n'assurent pas la couverture de la totalité du territoire national tant pour les adultes que les enfants. Pour ces derniers, les centres pratiquant l'ECMO sont peu nombreux, et les UMAC spécialisées encore plus rares.

Enfin, il est indispensable de prendre en considération les difficultés majeures que la France devrait affronter en cas de pandémie virale. Les capacités des centres accueillant les patients relevant de l'ECMO pourraient être vite dépassées, comme cela a été rapporté lors de l'épidémie de grippe H1N1 de 2009 [59]. Dans une telle situation, il est important qu'un certain nombre de centres puissent être rapidement opérationnels. L'organisation proposée doit ainsi permettre de faire face à une augmentation importante et rapide du nombre de patients à traiter en période épidémique.

Prenant en compte l'ensemble de ces commentaires, le jury de la conférence de consensus a fait les recommandations suivantes concernant les principes généraux de l'organisation au niveau national et au niveau des services de réanimation pour la prise en charge des patients souffrant de SDRA susceptibles de bénéficier du recours à l'ECMO.

\section{Principes généraux de l'organisation}

5.1 En regard de la qualité et de la sécurité des soins, une organisation territoriale structurée est indispensable à la prise en charge optimale des patients atteints de SDRA relevant de l'ECMO (RC).

5.2 En dehors de tout épisode épidémique, l'organisation sur le territoire national doit permettre la prise en charge d'au minimum 300 patients par an (RC).

5.3 La mise en place d'une activité d'ECMO doit s'inscrire dans un projet médical d'établissement (RC).

5.4 Il est nécessaire que, dans chaque région, soit identifié au minimum un centre référent possédant tous les moyens humains et matériels indispensables à la prise en charge d'un patient atteint de SDRA et à l'implantation et la conduite des techniques d'assistance extracorporelle : réanimation, chirurgie cardiaque et unité mobile d'assistance circulatoire (RC).

5.5 Les centres référents doivent disposer d'une unité mobile d'assistance circulatoire disponible 24 heures/24, à même d'intervenir dans tous les établissements de santé de la région concernée (RC).

Organisation des services de réanimation

5.6 Chaque service de réanimation doit colliger dans un registre national tous les patients pris en charge pour SDRA sévère (RC).

5.7 Chaque service de réanimation doit s'organiser par convention afin qu'au sein de chaque région la prise en charge des patients atteints de SDRA et relevant d'un traitement par ECMO soit assurée.

5.8 Dans chaque région, au moins un service de réanimation compétent pour l'ECMO, appartenant au centre référent ou ayant contractualisé avec l'un d'entre eux, doit être identifié (RC).

5.9 Un service de réanimation compétent pour la pratique de l'ECMO dans le SDRA doit obligatoirement : 1) acquérir et entretenir des compétences spécifiques ; 2) compter dans son personnel médical 
au minimum deux médecins formés ; 3) disposer d'un recours en urgence à la chirurgie vasculaire et thoracique ; 4) mettre en ouvre un programme régulier de formation du personnel paramédical ; 5) formaliser les indications et en assurer la traçabilité ; 6) renseigner le registre SDRA sévère ; 7) analyser, au moins une fois par an, à l'occasion de revues de morbidité-mortalité, l'ensemble des dossiers des patients traités par ECMO (RC).

5.10 Le maintien de la compétence en ECMO d'un service de réanimation risque de ne pas être assuré en deçà de dix indications annuelles d'ECMO.

\section{Spécificité de la prise en charge de l'enfant}

5.11 Pour l'enfant, une organisation interrégionale est nécessaire, organisée autour de centres référents pédiatriques (réanimation pédiatrique spécialisée), possédant tous les moyens humains et matériels indispensables à la prise en charge d'un patient atteint de SDRA et à l'implantation et la conduite des techniques d'assistance extracorporelle : réanimation, chirurgie cardiaque et unité mobile d'assistance circulatoire (RC).

5.12 Pour l'enfant, afin de permettre la réalisation de l'ECMO dans les meilleurs délais, le contact avec le centre référent pédiatrique (réanimation pédiatrique spécialisée) doit être précoce (RC).

\section{Perspectives}

Concernant les développements futurs, le jury de la conférence de consensus a souhaité faire les commentaires suivants :

- la grande méconnaissance à ce jour de l'épidémiologie du SDRA sévère en France constitue un obstacle majeur à la mise en place d'une stratégie argumentée d'utilisation des techniques d'assistance extracorporelle, et en particulier de l'ECMO. Le jury souhaite que soit mis en place rapidement le registre national des SDRA sévères pour tenter de répondre à cette interrogation ;

- la réalisation d'études multicentriques prospectives randomisées et la tenue de registres nationaux devraient permettre dans les années à venir de préciser les indications de l'ECMO VV dans la prise en charge du SDRA sévère ;

- le jury insiste sur l'absence d'indication reconnue à l'utilisation de l'ECCO $\mathrm{C}_{2}-\mathrm{R}$ et recommande de ne permettre son utilisation au cours du SDRA que dans le cadre de travaux de recherche clinique comportant tant des études multicentriques prospectives randomisées que des études de cohorte dûment constituées. Dans l'attente des résultats de ces études, une vigilance renforcée devrait s'exercer avant toute diffusion de nouveaux matériels disposant certes du marquage $\mathrm{CE}$, mais dont les rapports risque/ bénéfice et coût/bénéfice sont loin d'avoir été évalués ;

- enfin, les recommandations énoncées par le jury en ce qui concerne l'organisation proposée sont destinées à assurer une couverture nationale des besoins, aujourd'hui limités compte tenu des indications très réduites de l'ECMO $\mathrm{VV}$, mais aussi à créer un maillage national à même de faire face tant à un besoin accru épidémique type H1N1 qu'à un éventuel élargissement des indications fruit des résultats des essais en cours et de la mise à disposition prévisible de matériels et de consommables en permanente évolution.

Conflits d'intérêts : C. Richard, H. Outin et D. Osman sont co-investigateurs pour l'essai randomisé EOLIA (partenariat Maquet). L. Argaud, A. Blet, T. Boulain, L. Contentin, A. Dechartres, J.-M. Dejode, L. Donetti, M. Fartoukh, D. Fletcher, K. Kuteifan, S. Lasocki, J.-M. Liet, A.-C. Lukaszewicz, H. Mal, E. Maury, J.-C. Richard, F. Schneider, F. Tamion déclarent ne pas avoir de conflit d'intérêt.

\section{Références}

1. Ferguson ND, Fan E, Camporota L, et al (2012) The Berlin definition of ARDS: an expanded rationale, justification, and supplementary material. Intensive Care Med 38:1573-82

2. Slutsky AS, Ranieri VM (2013) Ventilator-induced lung injury. N Engl J Med 369:2126-36

3. Gattinoni L, Pesenti A, Mascheroni D, et al (1986) Low-frequency positive-pressure ventilation with extracorporeal $\mathrm{CO}_{2}$ removal in severe acute respiratory failure. JAMA 256:881-6

4. Morris AH, Wallace CJ, Menlove RL, et al (1994) Randomized clinical trial of pressure-controlled inverse ratio ventilation and extracorporeal $\mathrm{CO}_{2}$ removal for adult respiratory distress syndrome. Am J Respir Crit Care Med 149:295-305

5. Davies A, Jones D, Bailey M, et al (2009) Extracorporeal Membrane Oxygenation for 2009 Influenza A(H1N1) Acute Respiratory Distress Syndrome. JAMA 302:1888-95

6. Noah MA, Peek GJ, Finney SJ, et al (2011) Referral to an extracorporeal membrane oxygenation center and mortality among patients with severe 2009 influenza A(H1N1). JAMA 306:165968

7. Peek GJ, Mugford M, Tiruvoipati R, et al (2009) Efficacy and economic assessment of conventional ventilatory support versus extracorporeal membrane oxygenation for severe adult respiratory failure (CESAR): a multicentre randomized controlled trial. Lancet 374:1351-63

8. Pham T, Combes A, Roze H, et al (2013) Extracorporeal membrane oxygenation for pandemic influenza $\mathrm{A}(\mathrm{H} 1 \mathrm{~N} 1)$-induced acute respiratory distress syndrome: a cohort study and propensity-matched analysis. Am J Respir Crit Care Med 187:276-85

9. Brodie D, Bacchetta M (2011) Extracorporeal membrane oxygenation for ARDS in adults. N Engl J Med 365:1905-14

10. Ranieri VM, Rubenfeld GD, Thompson BT, et al (2012) Acute respiratory distress syndrome: the Berlin Definition. JAMA 307:2526-33 
11. Abrams D, Brodie D (2013) Emerging indications for extracorporeal membrane oxygenation in adults with respiratory failure. Ann Am Thorac Soc 10:371-7

12. Bein T, Weber-Carstens S, Goldmann A, et al (2013) Lower tidal volume strategy (approximately $3 \mathrm{ml} / \mathrm{kg}$ ) combined with extracorporeal $\mathrm{CO}_{2}$ removal versus 'conventional' protective ventilation $(6 \mathrm{ml} / \mathrm{kg})$ in severe ARDS: the prospective randomized Xtravent-study. Intensive Care Med 39:847-56

13. Atkins D, Best D, Briss PA, et al (2004) Grading quality of evidence and strength of recommendations. BMJ 328:1490

14. Guyatt GH, Oxman AD, Vist GE, et al (2008) GRADE: an emerging consensus on rating quality of evidence and strength of recommendations. BMJ 336:924-6

15. Abrams D, Brodie D, Combes A (2013) What is new in extracorporeal membrane oxygenation for ARDS in adults? Intensive Care Med 39:2028-30

16. Combes A, Bacchetta M, Brodie D, et al (2012) Extracorporeal membrane oxygenation for respiratory failure in adults. Curr Opin Crit Care 18:99-104

17. Gattinoni L, Carlesso E, Langer T (2011) Clinical review: Extracorporeal membrane oxygenation. Crit Care 15:243

18. Sidebotham D, Allen SJ, McGeorge A, et al (2012) Venovenous extracorporeal membrane oxygenation in adults: practical aspects of circuits, cannulae, and procedures. J Cardiothorac Vasc Anesth 26:893-909

19. Navia JL, Atik FA, Beyer EA, Ruda Vega P (2005) Extracorporeal membrane oxygenation with right axillary artery perfusion. Ann Thorac Surg 79:2163-5

20. Bein T, Weber F, Philipp A, et al (2006) A new pumpless extracorporeal interventional lung assist in critical hypoxemia/hypercapnia. Crit Care Med 34:1372-7

21. Cove ME, Maclaren G, Federspiel WJ, Kellum JA (2012) Bench to bedside review: Extracorporeal carbon dioxide removal, past present and future. Crit Care 16:232

22. Sidebotham D, McGeorge A, McGuinness S, et al (2010) Extracorporeal membrane oxygenation for treating severe cardiac and respiratory failure in adults: part 2-technical considerations. J Cardiothorac Vasc Anesth 24:164-72

23. Schmidt M, Zogheib E, Rozé H, et al (2013) The PRESERVE mortality risk score and analysis of long-term outcomes after extracorporeal membrane oxygenation for severe acute respiratory distress syndrome. Intensive Care Med 39:1704-13

24. Hirose H, Yamane K, Marhefka G, Cavarocchi N (2012) Right ventricular rupture and tamponade caused by malposition of the Avalon cannula for venovenous extracorporeal membrane oxygenation. J Cardiothorac Surg 7:36

25. 2013 ELSO Guidelines for Cardiopulmonary Extracorporeal Life Support, Extracorporeal Life Support Organisation. www.elsonet.org

26. Ensminger SM, Puehler T, Benzinger M, Morshuis M, Kizner L, Gummert JF (2012) The role of extracorporeal mechanical assists. Applied Cardiopulmonary Pathophysiology 16:192-201

27. Toomasian JM, Bartlett RH (2011) Hemolysis and ECMO pumps in the 21st Century. Perfusion 26:5-6

28. Del Sorbo LCM, Fan E (Published online October 17, 2013) Extracorporeal life support for adults with severe acute respiratory failure. Lancet Respir www.thelancet.com/respiratory. http://dx.doi.org/10.1016/S2213-2600(13)70197-8

29. Kohler K, Valchanov K, Nias G, Vuylsteke A (2013) ECMO cannula review. Perfusion 28:114-24

30. Lequier L, Horton SB, McMullan DM, Bartlett RH (2013) Extracorporeal membrane oxygenation circuitry. Pediatr Crit Care Med $14:$ S7-S12

31. Leger PL, Guilbert J, Isambert S, et al (2013) Pediatric singlelumen cannula venovenous extracorporeal membrane oxygenation: a French center experience. Artif Organs 37:57-65
32. Guerin C, Reignier J, Richard JC, et al (2013) Prone positioning in severe acute respiratory distress syndrome. N Engl J Med 368:2159-68

33. Khemani RG, Newth CJ (2010) The design of future pediatric mechanical ventilation trials for acute lung injury. Am J Respir Crit Care Med 182:1465-74

34. Zapol WM, Snider MT, Hill JD, et al (1979) Extracorporeal membrane oxygenation in severe acute respiratory failure. A randomized prospective study. JAMA 242:2193-6

35. Miller RR 3rd, Markewitz BA, Rolfs RT, et al (2013) Clinical findings and demographic factors associated with ICU admission in Utah due to novel 2009 influenza A(H1N1) infection. Chest 137:752-8

36. Brechot N, Luyt CE, Schmidt M, et al (2013) Venoarterial extracorporeal membrane oxygenation support for refractory cardiovascular dysfunction during severe bacterial septic shock. Crit Care Med 41:1616-26

37. Borchard A, Schwappach DL, Barbir A, Bezzola P (2012) A systematic review of the effectiveness, compliance, and critical factors for implementation of safety checklists in surgery. Ann Surg 256:925-33

38. Ball RD, Scouras NE, Orebaugh S, et al (2012) Randomized, prospective, observational simulation study comparing residents' needle-guided vs free-hand ultrasound techniques for central venous catheter access. Br J Anaesth 108:72-9

39. Ranasinghe AM, Peek GJ, Roberts N, et al (2004) The use of transesophageal echocardiography to demonstrate obstruction of venous drainage cannula during ECMO. ASAIO J 50:619-20

40. Langer T, Vecchi V, Belenkiy SM, et al (2013) Pressure-guided positioning of bicaval dual-lumen catheters for venovenous extracorporeal gas exchange. Intensive Care Med 39:151-4

41. Trimlett RH, Cordingley JJ, Griffiths MJ, et al (2011) A modified technique for insertion of dual lumen bicaval cannulae for venovenous extracorporeal membrane oxygenation. Intensive Care Med 37:1036-7

42. Paden ML, Conrad SA, Rycus PT, Thiagarajan RR (2013) Extracorporeal Life Support Organization Registry Report 2012. ASAIO J 59:202-10

43. Koster A, Dyke CM, Aldea G, et al (2007) Bivalirudin during cardiopulmonary bypass in patients with previous or acute heparin-induced thrombocytopenia and heparin antibodies: results of the CHOOSE-ON trial. Ann Thorac Surg 83:572-7

44. Yuan S, Tsukahara E, De La Cruz K, Kelly RB (2013) How we provide transfusion support for neonatal and pediatric patients on extracorporeal membrane oxygenation. Transfusion 53:1157-65

45. Hoover NG, Heard M, Reid C, et al (2008) Enhanced fluid management with continuous venovenous hemofiltration in pediatric respiratory failure patients receiving extracorporeal membrane oxygenation support. Intensive Care Med 34:2241-7

46. Ejike JC, Schenkman KA, Seidel K, et al (2006) Cerebral oxygenation in neonatal and pediatric patients during veno-arterial extracorporeal life support. Pediatr Crit Care Med 7:154-8

47. Bembea MM, Annich G, Rycus P, et al (2013) Variability in anticoagulation management of patients on extracorporeal membrane oxygenation: an international survey. Pediatr Crit Care Med 14:e77-e84

48. Rollins MD, Hubbard A, Zabrocki L, et al (2012) Extracorporeal membrane oxygenation cannulation trends for pediatric respiratory failure and central nervous system injury. J Pediatr Surg 47:68-75

49. Haefner SM, Bratton SL, Annich GM, et al (2003) Complications of intermittent prone positioning in pediatric patients receiving extracorporeal membrane oxygenation for respiratory failure. Chest 123:1589-94 
50. Mac Laren G, Combes A, Bartlett RH (2012) Contemporary extracorporeal membrane oxygenation for adult respiratory failure: life support in the new era. Intensive Care Med 38:210-20

51. Marasco SF, Lukas G, McDonald M, et al (2008) Review of ECMO (extra corporeal membrane oxygenation) support in critically ill adult patients. Heart Lung Circ 17:S41-S7

52. Roch A, Lepaul-Ercole R, Grisoli D, et al (2010) Extracorporeal membrane oxygenation for severe influenza $A(H 1 N 1)$ acute respiratory distress syndrome: a prospective observational comparative study. Intensive Care Med 36:1899-905

53. Ullrich R, Lorber C, Roder G, et al (1999) Controlled airway pressure therapy, nitric oxide inhalation, prone position, and extracorporeal membrane oxygenation (ECMO) as components of an integrated approach to ARDS. Anesthesiology 91:1577-86

54. Freeman CL, Bennett TD, Casper TC, et al (2014) Pediatric and neonatal extracorporeal membrane oxygenation: does center volume impact mortality? Crit Care Med 42:512-9
55. Karamlou T, Vafaeezadeh M, Parrish AM, et al (2013) Increased extracorporeal membrane oxygenation center case volume is associated with improved extracorporeal membrane oxygenation survival among pediatric patients. J Thorac Cardiovasc Surg $145: 470-5$

56. Forrest P, Ratchford J, Burns B, et al (2011) Retrieval of critically ill adults using extracorporeal membrane oxygenation: an Australian experience. Intensive Care Med 37:824-30

57. Roch A, Hraiech S, Masson E, et al (2013) Outcome of acute respiratory distress syndrome patients treated with extracorporeal membrane oxygenation and brought to a referral center. Intensive Care Med 40:74-83

58. Cabrera AG, Prodhan P, Cleves MA, et al (2011) Interhospital transport of children requiring extracorporeal membrane oxygenation support for cardiac dysfunction. Congenit Heart Dis 6:202-8

59. Richard JC, Pham T, Brun-Buisson C, et al (2012) Interest of a simple on-line screening registry for measuring ICU burden related to an influenza pandemic. Crit Care 16:R118 